\title{
Recovery of 3-D shape from deforming contours
}

\author{
JAMES M. CORTESE and GEORGE J. ANDERSEN \\ University of Illinois, Urbana-Champaign, Champaign, Illinois
}

\begin{abstract}
Three experiments were conducted to examine the accuracy of 3-D shape recovery from deformingcontour displays. The displays simulated silhouettes of ellipsoids rotating about a vertical axis. Subjects judged the horizontal cross-section of the ellipsoids. The shape of the ellipsoid, the position of the axis of rotation, and the type of projection were manipulated in Experiment 1 . The results indicated relatively accurate shape recovery when the major axis of the ellipsoid was small. In Experiment 2, the shape of the ellipsoid and the velocity and curvature of the contour were manipulated. When the rate of deformation of curvature was decreased, more eccentric shapes were reported. In Experiment 3, the shape of the object and the amount of simulated rotation were manipulated. Subjects made both shape and extent of rotation judgments. The results showed that eccentricity of shape responses could be accurately predicted from rotation responses, suggesting that the recovery of 3-D shape from smooth, deforming contours is dependent on the perceived extent of rotation.
\end{abstract}

Elaborating on the early work of Miles (1931) and Metzger (1934), Wallach and O'Connell (1953) demonstrated that 3-D shape could be recovered from orthographic projections of rotating objects. In one condition, a solid, truncated cylinder was rotated. The resulting image contour deformed over time in one dimension only, and subjects reported a perception of a nonrigid, 2-D object. However, when bent wire-frame figures or planarsurfaced solids were rotated, the contour simultaneously deformed in both the vertical and the horizontal dimensions. The subjects in these conditions reported a perception of a rotating, 3-D object. Wallach and O'Connell proposed the term kinetic depth effect for this phenomenon and suggested that contour length and direction changes were important for the perception of 3-D shape.

More recently, researchers have examined the minimal conditions for the recovery of 3-D shape from motion. For example, Ullman (1979) showed that, for a rigid configuration, three orthographic views of four noncoplanar points were sufficient for the recovery of the 3-D structure. In recent studies, researchers have examined the validity of these models for human perception with the use of computer-generated displays in which points are depicted on a 3-D object. Such displays have been used to examine the importance of specific constraints (Braunstein \& Andersen, 1984; Todd, 1984) and of minimal conditions (Braunstein, Hoffman, \& Pollick, 1990; Braunstein, Hoffman, Shapiro, Andersen, \& Bennett, 1987; Lappin, Doner, \& Kottas, 1980) proposed in computational models of the perception of 3-D shape.

This research was supported by a National Science Foundation Graduate Fellowship awarded to James M. Cortese and National Science Foundation Grant BNS 8908512. The authors would like to thank Myron L. Braunstein, Bart De Bruyn, James T. Todd, and an anonymous reviewer for comments on an earlier draft of this manuscript. Reprint requests should be sent to George J. Andersen, Department of Psychology, University of California, Riverside, CA 92521.
An important characteristic of both the dot and the deforming contour displays is the presence of image features that always correspond, over time, to the same points on the 3-D object. In dot displays, the texture elements in the image always correspond to the same texture elements on the 3-D structure. The contour displays used by Wallach and O'Connell (1953) also have motion correspondence, because a wire-figure or planar-surfaced solid will produce an image contour in correspondence with fixed points on the object. The availability of motion correspondence has been an implicit assumption in many structure-from-motion proofs (see Todd, 1985, for a discussion of this issue).

In contrast to the contour displays used by Wallach and O'Connell (1953), contour displays of rotating, smooth objects have in general no motion correspondence. During rotation of a smooth solid object, the image contour deforms, and points on the contour do not map, over time, to the same points on the object itself. Todd (1985) used displays of this type to examine the necessity of motion correspondence for 3-D shape recovery. In Todd's demonstration, subjects were shown orthographic projections of opaque ellipsoids rotating about a vertical axis (an ellipsoid is a quadric surface defined by three mutually orthogonal axes-see Figure 1). No texture was present on the surface; only a silhouette of the ellipsoid appeared in the image. When a single ellipsoid was shown, subjects reported a deforming 2-D shape. However, when two ellipsoids moving in phase were shown, the subjects reported a perception of a rotating 3-D object. Todd concluded that motion correspondence was not necessary for the perception of 3-D shape from deforming contours.

Todd's demonstration indicates that 3-D form can be recovered from the contour deformation produced by rotating objects. Although several current theories have suggested that contours are important for the recovery and recognition of 3-D shape (Biederman, 1985; Hoffman \& 
A.

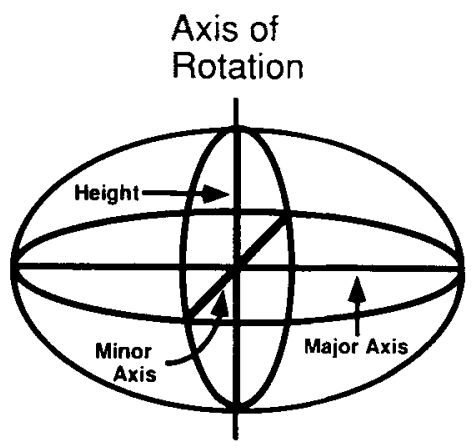

B. Axis of

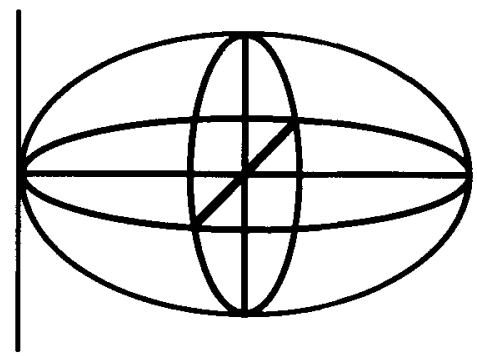

Figure 1. An ellipsoid is defined by three mutually orthogonal axes, which for convenience are referred to as "major axis," "minor axis," and "height." (A) Centered rotation condition. (B) Off-centeredrotation condition.

Richards, 1984; Koenderink, 1984), there has been no investigation of the specific information used for the recovery of 3-D shape from deforming contours. Our purpose in the present study was to examine the accuracy with which subjects could recover 3-D shape from deforming contours, and to determine the specific information used for the recovery of shape.

The problem of 3-D shape recovery is not trivial, because the projected silhouette of a rotating smooth object is fundamentally ambiguous. Consider an orthographic projection of an ellipsoid rotating about a vertical axis. Without constraints such as rigidity and constant angular velocity, there exist an infinite set of transformations of objects that are consistent with the projected deformation of a given rotating ellipsoid (see Figure 2). In order for subjects to recover the 3-D shape, constraints must be imposed that restrict the interpretation of image information.

Our purpose in the present study was to determine what information is used for the recovery of 3-D shape from deforming contours. Possible sources of information that the human visual system might use include:

1. Contour deformation. In general, as a solid 3-D object rotates, the projected image contour deforms over time. In the computer vision literature, mathematical proofs have been reported for the reconstruction of surfaces from contour deformation. Giblin and Weiss (1987) have demonstrated that smooth surfaces can be reconstructed from a series of orthographic projections, provided that the viewing directions are coplanar. Blake and
Cipolla (1989) have extended these theories to general nonplanar viewing directions and perspective projection.

2 . Motion correspondence. Under orthographic projection, motion correspondence does exist for two points on the contour of an ellipsoid, assuming rotation about a single axis. When an ellipsoid rotates about a vertical axis, for example, the extreme vertical points on the contour map directly to the extreme vertical points on the surface. If the axis of rotation is coincident with an axis of the ellipsoid, these points will be stationary. However, if the axis of rotation is not coincident with the axis of the ellipsoid, these points will move sinusoidally. Pollick (1989) has suggested that the sinusoidal motion of these points could be used to derive angular velocity, which in turn might be useful in the recovery of 3-D shape.

Although these sources of information were available in the displays used by Todd (1985), Todd's subjects did not report a compelling 3-D perception when a single ellipsoid was presented. We conducted a pilot study to determine whether a single ellipsoid could produce a compelling perception of a rotating 3-D object. Subjects viewed a display simulating rotation of an opaque nontextured ellipsoid positioned in front of a static randomly textured background. The contour of the ellipsoid was defined only through occlusion of the background texture elements (see Figure 3). Occlusion was used throughout these experiments because it is a useful source of information for specifying the 2-D contour of objects (Andersen \& Cortese, 1989). In addition, Kaplan (1969) showed

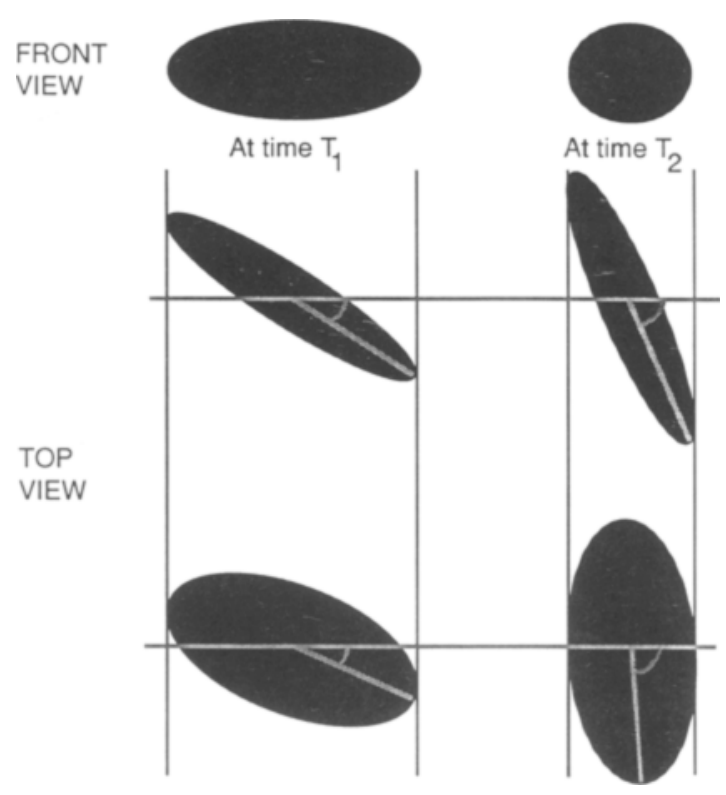

Figure 2. The ambiguity of the projected image. The front view depicts the projected image of a rotating ellipsoid at two positions $\left(T_{1}\right.$ and $\left.T_{2}\right)$. The top view depicts two of the infinitely many possible 3-D objects whose projections are consistent with the same image transformation. The position of the major axis is shown in the top view to indicate that different amounts of rotation have occurred for the two objects. 


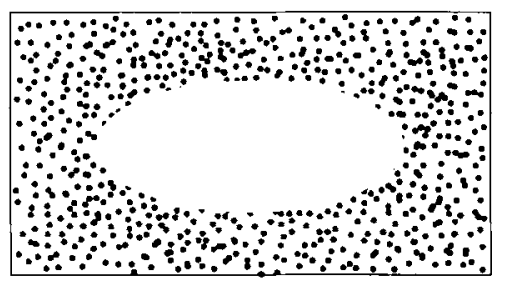

Frame 1

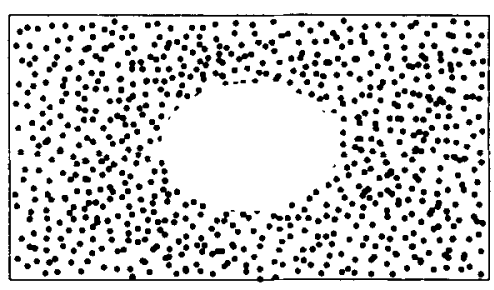

Frame 2

Figure 3. Two frames illustrating the type of stimuli used in the present experiments. The frames simulate rotation of an opaque nontextured ellipsoid positioned in front of a static random-textured background.

that occlusion can be used for the recovery of depth order. Informal observations suggested that the perception of separate surfaces in depth produced a more salient perception of a 3-D object in the foreground.

The ellipsoid had a vertical axis of $4.2^{\circ}$ of visual angle. The two horizontal axes were $6.8^{\circ}$ and $2.0^{\circ}$. The ellipsoid underwent a continuous $360^{\circ}$ rotation about a vertical axis tangent to the end point of the object (see Figure 1B). The subjects viewed the displays without instruction, and they were asked, after several seconds, to describe what they saw. All 12 naive observers reported a perception of a rotating 3-D object. Some of the observers reported that the object looked like a football or a watermelon. These informal observations suggest that subjects can see a compelling 3-D shape from rotation of a single ellipsoid. The apparent increased saliency of the perception of 3-D shape, compared to Todd's (1985) results, could be due to our use of occlusion, or to the different position of the axis of rotation (off-centered rather than centered rotation), or to both factors. On the basis of these informal results, we presented subjects with displays simulating rotation of a single ellipsoid in all of our experiments.

\section{EXPERIMENT 1}

In our pilot study, and in certain conditions studied by Todd (1985), subjects reported a compelling kinetic depth effect. Our purpose in Experiment 1 was to determine how accurately observers could recover 3-D shape from deforming contours. We examined this issue by requiring subjects to judge the cross-section of rotating ellipsoids.
The displays used in our pilot study and in Todd's demonstration consisted of simulated ellipsoids rotating $360^{\circ}$ about a vertical axis. It is important to note that if a complete $360^{\circ}$ rotation were used in the present experiments, there would be 2-D information sufficient to specify all three axes. If the projection is orthographic, an axis is specified in the 2-D projection whenever it is perpendicular to the line of sight, and thus a rotation of as little as $90^{\circ}$ could provide this 2-D information. For rotation about a vertical axis, the maximum and minimum horizontal extents of the image contour provide two of the axes, and the vertical extent provides the third. Thus, subjects would merely have to identify the maximum and minimum horizontal extents of the projected deforming contour to identify the horizontal cross-section. In order to restrict this 2-D information, and in order to provide an approximate measure of the extent to which the subjects used this information, the angle of rotation was restricted to $63^{\circ}$ within a single quadrant. If the subjects now assumed that the maximum and minimum horizontal extents represent the two horizontal axes, their responses would be inaccurate. To check this possibility, the responses of a hypothetical subject, using only the extreme horizontal extents in the image contour, were determined. In the text and figures, we will refer to these hypothetical responses as the $2-D$ cue line.

An additional issue examined in Experiment 1 was the importance of motion-correspondence points for the recovery of 3-D shape from deforming contours. As discussed above, the extreme vertical points on the image contour produced by a rotating ellipsoid specify corresponding points on the object. To examine the importance of this information, we manipulated two variables: the type of rotation (about an axis coincident or noncoincident with the vertical axis of the ellipsoid), and the type of projection (orthographic or perspective). Consider a display simulating an ellipsoid rotating, under orthographic projection, about a vertical axis. If the axis of rotation is not coincident with the vertical axis of the ellipsoid, the extreme vertical points of the image contour (which correspond, over time, to the same points on the object) will move sinusoidally. These points potentially provide information for angular velocity (Pollick, 1989). If, however, the axis of rotation is coincident with the vertical axis of the ellipsoid, these points will be stationary, and no points along the contour will provide sinusoidal velocity information. In the present study, subjects viewed displays simulating ellipsoids rotating about a vertical axis that was either coincident (centered) or noncoincident (off-centered) with the vertical axis defining the ellipsoid (see Figure 1).

A second method for varying the presence of corresponding points is to include both orthographic and perspective projection conditions. Under orthographic projection, the image contour will always have two corresponding points (assuming rotation about a single axis). However, under perspective projection, there are no corresponding points 
in the image contour. Consider the curve that divides the visible portions of the surface from the occluded portions. This is the 3-D space curve that Koenderink (1984) has called the rim. Under perspective projection, the rim, even at the extreme vertical points, moves over the object during rotation as portions of its surface approach or recede. Thus, the extreme vertical points in a perspective projection do not correspond to the extreme vertical points on the surface, but rather to points closer to the viewer. Therefore, the variation in the type of projection provides another method for examining the importance of motioncorrespondence points.

\section{Method}

Subjects. The subjects were 16 students from the University of Illinois who received course credit for their participation. All subjects were naive concerning the purpose of the experiment, and all had normal or corrected-to-normal vision.

Design. Four independent variables were examined: type of projection (perspective or orthographic), location of the axis of rotation (centered or off-centered), the larger horizontal axis of the ellipsoid, and the smaller horizontal axis of the ellipsoid.

Stimuli. As depicted in Figure 1, an ellipsoid is completely defined by three mutually orthogonal axes. Because the ellipsoids in these experiments were all presented in the same orientation, for convenience the term major axis will be used to refer to the larger of the two axes that define the horizontal cross-section, and the term minor axis will indicate the smaller. The displays simulated silhouettes of ellipsoids occluding a background of random dots. Thus, the contour of the ellipsoid was specified by the occlusion of background texture elements. One thousand texture elements were randomly positioned in a background of approximately $237.4 \mathrm{~cm}^{2}$, resulting in a density of $4.2 \mathrm{dots} / \mathrm{cm}^{2}$. At a viewing distance of $1.2 \mathrm{~m}$, the background was $9.0^{\circ} \times 5.8^{\circ}$ of visual angle, which resulted in a texture density of 19.2 dots $/ \mathrm{deg}^{2}$. All ellipsoids were presented with the major axis horizontal and were depicted rotating about a vertical axis. The axis of rotation intersected the center of the ellipsoid for the centered rotation condition, whereas the axis intersected the horizontal end point for the off-centered rotation condition. The ellipsoids began with the major axis at an angle $9^{\circ}$ from the image plane (perpendicular to the line of sight) and rotated to a position $72^{\circ}$ from the image plane. The ellipsoids oscillated three times between these two points. The rate of rotation was $4.5^{\circ} /$ frame, and the frame duration was $70 \mathrm{msec}$, resulting in a rate of rotation of $10.7 \mathrm{rpm}$. There were 15 frames in the displays, and the total display duration was $6.3 \mathrm{sec}$.

The extent of the major axis in the large major axis condition was twice the extent in the small major axis condition. The size of the projected contour changed as the objects rotated. The ellipsoids in the larger major axis condition filled approximately $7.0^{\circ}$ of visual angle at their greatest horizontal extent, and $2.4^{\circ}$ at their least horizontal extent (for the smallest level of the minor axis). Those in the smaller major axis condition were approximately $3.5^{\circ}$ at their greatest extent and $2.4^{\circ}$ at their least extent. The height of the objects was approximately $3.0^{\circ}$ visual angle. The minor axis to major axis ratios for the larger major axis with the four levels of the minor axis were $.12, .20, .28$, and .36 (see Table 1). With the smaller major axis, at one half the size of the larger, the ratios were twice these values, at $.24, .40, .56$, and .72 . The ratio of the major axis to the third axis (height) was 1.2 to 1 for the smaller major axis, and 2.3 to 1 for the larger.

The perspective ratio (the distance between the projection point and the farthest point of the object divided by the distance between the projection point and the closest point of the object) varied for the different shape conditions. The ratios were $1.06,1.13,1.07$,
Table 1

Minor Axis to Major Axis Ratios

\begin{tabular}{ccccr}
\hline \multicolumn{5}{c}{ Experiment 1 } \\
\hline & \multicolumn{4}{c}{ Minor Axis } \\
Major Axis & 36 & 60 & 84 & 108 \\
\hline 150 & 0.24 & 0.40 & 0.56 & 0.72 \\
300 & 0.12 & 0.20 & 0.28 & 0.36 \\
\hline
\end{tabular}

Experiment 2

\begin{tabular}{cccc}
\hline & \multicolumn{3}{c}{ Minor Axis } \\
\cline { 2 - 4 } Major Axis & 40 & 80 & 160 \\
\hline 250 & 0.16 & 0.32 & 0.64 \\
500 & 0.08 & 0.16 & 0.32 \\
\hline
\end{tabular}

\begin{tabular}{crrrr}
\hline \multicolumn{5}{c}{ Experiment 3 } \\
\cline { 2 - 5 } Minor Axis & 139.22 & 179.00 & 250.60 & 358.00 \\
\hline 100.24 & 0.72 & 0.56 & 0.40 & 0.28 \\
\hline
\end{tabular}

Note-Axis lengths are given in screen units.

and 1.14 for the centered rotation short major axis, centered rotation long major axis, off-centered rotation short major axis, and off-centered rotation long major axis, respectively. The viewing distance was appropriate for the simulated geometry of the object, projection point, and textured background surface.

Apparatus. The stimuli were displayed on a Hewlett-Packard Model 1310B display scope with a p-31 phosphor, under the control of a PDP-11/73 computer. A digital-to-analog board that was designed and constructed at the University of Illinois was used to present points on the display scope. The subjects viewed the displays monocularly, through a tube arrangement that limited the field of view to $11.7^{\circ}$ of visual angle. The subjects viewed the displays from a distance of $1.2 \mathrm{~m}$.

Styrofoam models of some of the shapes to be depicted were used for demonstration. These models included a sphere, two different ellipsoids, and a flat cutout of an ellipse. In addition, a chart (for making the shape responses) was used which showed 26 ellipses with minor to major axis ratios ranging from 0 (a straight line) to 1 (a circle), with the ratios increasing in steps of .04 (see Figure 4).

Procedure. The subjects were instructed that they would see a series of displays of moving objects. They were not told that the objects would be rotating. The subjects were instructed that the displays might appear to be somewhat spherical, egg-shaped, or flat. These possibilities were demonstrated with the models. For each display, the subjects rated the amount of perceived depth on an 11point scale, with 0 representing no perceived depth, and 10 representing a strong impression of depth. Also for each display, the subjects made shape judgments on a 26-point scale, utilizing the chart described above and depicted in Figure 4. The subjects were told that these ellipses represented a top view of the objects that they would see. The subjects were told to imagine how the shape of the object would appear if they were looking down on it from above. Thus the subjects were told to report the shape of the object in horizontal cross-section. If the display looked completely flat, subjects were to choose number 1 , the straight line. If it looked perfectly spherical, they were to choose number 26 , the circle. The subjects were given 20 min of random practice trials followed by 3 presentations of each display condition presented in a random order.

At the end of the experiment, the subjects were asked to describe what the objects were doing. They were also asked if they had any difficulty seeing the contours of the objects, and they were asked if they used any particular strategy to make the shape judgments. 


\section{Results and Discussion}

During debriefing, the subjects typically reported that the objects appeared to be spinning back and forth. Four of the 16 subjects, however, reported that the objects in many displays appeared to stretch and shrink. These 4 subjects apparently saw 2-D nonrigid motion, and not rigid rotation, in many of the displays. These subjects were relatively less accurate in estimating the cross-sections of the rotating ellipsoids. Their mean error in shape responses was 6.7 on the 26-point scale, compared to 3.5 for those subjects reporting a perception of rotation. No further analysis was done on the responses of these 4 subjects. The data below are for the 12 subjects who spontaneously reported rotation.

The subjects' shape and depth responses were analyzed in four-way analyses of variance (ANOVAs). The shape responses showed a significant effect for the minor axis $[F(3,33)=58.2, p<.001]$, which was expected, since variations in the minor axis affected the shape of the object. There was a significant interaction between the major and the minor axis $[F(3,33)=19.0, p<.001]$. The $\omega^{2}$ values for these two significant results were .20 and .08 , respectively.

What is important to the issue of shape recovery, of course, is not significant effects per se but rather accuracy of shape recovery. In Figure 5, the shape responses from Experiment 1 are shown. It is clear from inspection of the figure that very good accuracy was obtained for the short major axis condition in comparison with the long major axis condition. In order to confirm this, a $t$ test was conducted in which the mean errors for the short and long major axis conditions were compared. The result of this

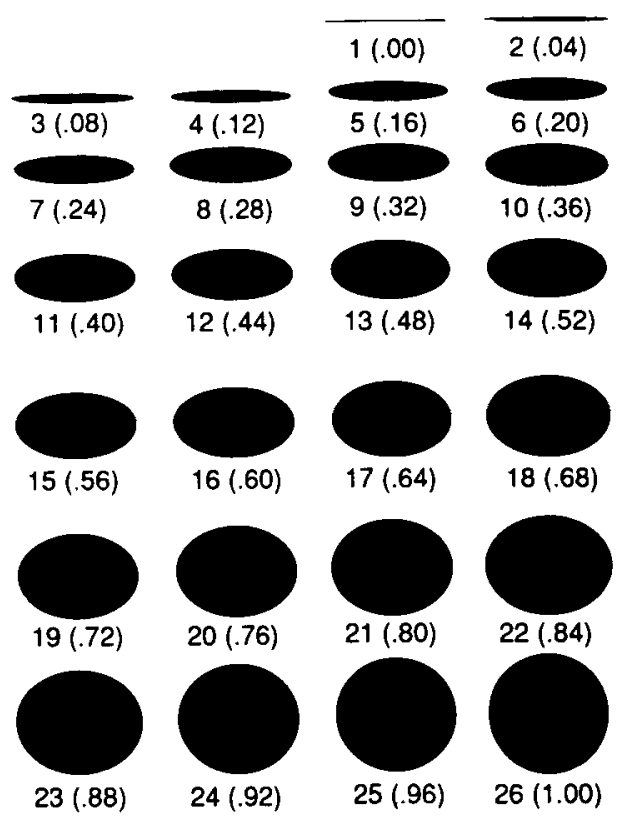

Figure 4. The chart used by the subjects to make shape responses. Minor axis to major axis ratios indicated here in parentheses were not present on the chart shown to subjects.

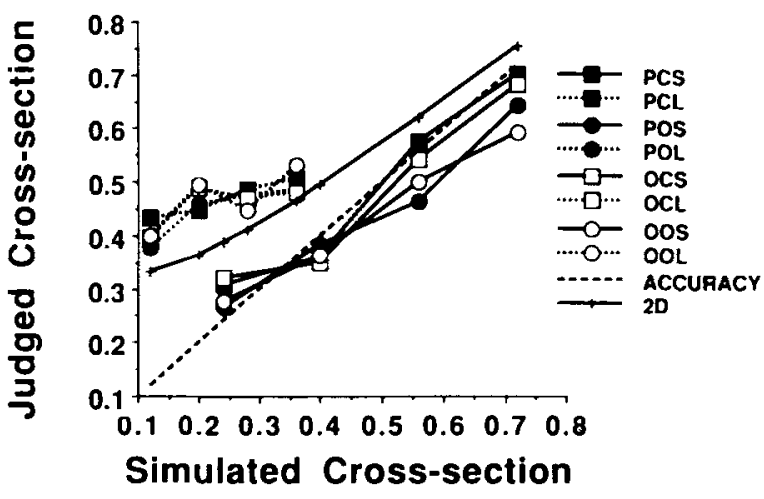

Figure 5. Mean shape judgments as a function of simulated crossection (minor axis to major axis ratio). The first character in the legend refers to perspective $(P)$ or orthographic $(O)$ projection. The second character refers to centered $(C)$ or off-centered $(O)$ rotation (see text). The third character refers to a long (L) or short (S) major axis. See text for an explanation of the 2-D cue line. The dashed line represents perfect performance.

test was significant $[t(15)=9.3, p<.001]$. The longer major axis condition consistently produced less accurate shape responses. The subjects reported ellipsoids that were less elongated (more nearly spherical) than those actually depicted. Notice that this is not merely a function of the eccentricity of the ellipsoid. The two sets of curves overlap in one region of the graph (with cross-section ratios from .24 to .36 ). In this region, the ellipsoids in the two major axis conditions have similar eccentricities. The upper set of curves, however, represents ellipsoids that have twice the major and minor axes of the smaller (the third axis, the height of the ellipsoid, was the same throughout Experiment 1). The fact that equivalent levels of accuracy were not obtained for the two sets of curves indicates that eccentricity is not the explanation for the differences across major axis conditions. Furthermore, the 2-D cue line, which represents the minimum horizontal extent in a given display divided by the maximum horizontal extent, reveals that the good accuracy obtained in the smaller major axis condition did not result merely from the use of the 2-D information in the displays. Had subjects used this information, their estimates of the cross-section would have been less eccentric (more circular).

Analysis of the depth responses, depicted in Figure 6, revealed a significant effect for the major axis condition $\left[F(1,11)=14.1, p<.005, \omega^{2}=.10\right]$. The larger major axis produced higher depth ratings from the subjects, despite the fact that in this condition there was poorer shape recovery. Indeed, a correlation between the depth responses and the mean errors in each condition revealed a significant positive relationship $[R(30)=.72, p<$ $.001]$. The subjects were less accurate in conditions in which higher depth ratings were obtained. As the major axis of the ellipsoid was increased, the extent of the object in depth also increased. As indicated above, the larger major axis produced higher depth ratings and less accurate shape responses (more circular cross-section estimates), 


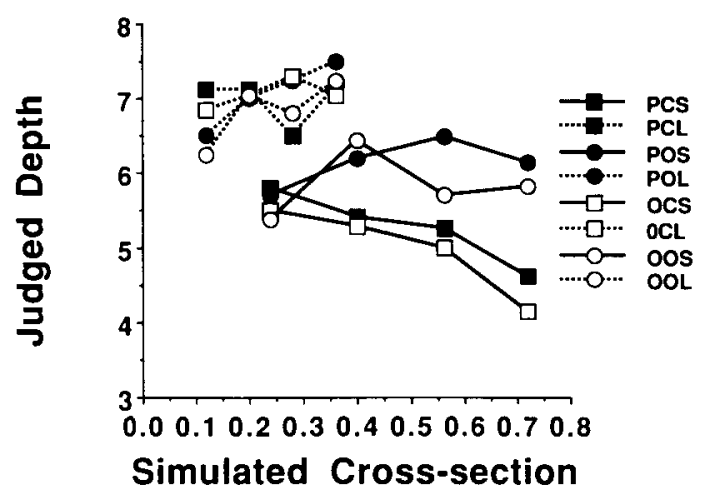

Figure 6. Mean depth ratings as a function of simulated crosssection (minor axis to major axis ratio). The first character in the legend refers to perspective $(P)$ or orthographic $(O)$ projection. The second character refers to centered $(C)$ or off-centered $(O)$ rotation (see text). The third character refers to a long (L) or short (S) major axis.

in comparison with the depth and shape responses for the shorter major axis. Because the depth response is likely to be influenced by the extent of the major axis, this result may suggest that the poor accuracy of shape recovery (more circular cross-section estimates) for the longer major axis condition resulted from an overestimation of the minor axis, rather than an underestimation of the major axis.

The depth responses also showed a significant interaction between the minor axis and the axis location $[F(3,33)$ $\left.=4.5, p<.025, \omega^{2}=.01\right]$. This interaction can be seen most clearly with the set of curves from the shorter major axis condition in Figure 6. As the depicted shapes increase on the scale (representing more nearly circular crosssections), depth ratings increase for off-centered rotations, but they decrease for centered rotations. This result is not merely a function of the simulated extent of depth, because within a major axis condition the greatest extent in depth is constant.

Other than this interactive effect for depth ratings, the axis location variable had no significant effect on subjects' responses. It is not surprising that this variable did not affect shape judgments, because the contour deformation is the same for an orthographic projection, and it is very similar for a perspective projection. It was surprising, however, that the variable did not have a stronger effect on depth ratings, since informal observations had suggested that the off-centered rotation produced a more salient depth perception.

The lack of a significant effect of axis location on the shape judgments suggests that there was no advantage to having the two moving points of correspondence (the top and bottom of the contour) that are present in the offcentered case. This suggests that sinusoidal angular velocity may not be used for the recovery of 3-D shape from deforming contours. Note, however, that these two points of correspondence, for these occlusion displays, are only present on a subjective contour and may have been difficult to identify.

Finally, the effect of perspective as opposed to orthographic projection was not significant $[F(1,11)=4.3$, $p>.05]$. There were no other significant effects or interactions.

The main result of Experiment 1 was that subjects who reported a perception of rotation in depth had good accuracy in the shorter major axis condition, and poor accuracy in the longer major axis condition. This result was consistent across variations in the projection and axis location variables. A possible explanation for this result is that for the longer major axis condition the image velocity and rate of curvature change were too great. If the major axis of the ellipsoid is increased, with a given angular velocity and with the other axes constant, both the image velocity and the curvature (along the right and left edges of the image contour) will increase. The maximum image velocity increased as the major axis increased, and it decreased as the minor axis increased.

The effect of curvature variation was analyzed by considering the curvature of the (left and right) ends of the contour for each frame depicted in the display. A significant correlation was found between the mean error in each condition and the variance of these curvature values $[r(6)$ $=.94, p<.001]$. The subjects were highly accurate in the conditions in which the variance of the curvature values was relatively small. As mentioned earlier, either an increase in rate of change of curvature or an increase in image velocity could potentially impair the shaperecovery process. In order to test these possibilities, we manipulated the angular velocity, which obviously affects the image velocity, in Experiment 2 . In addition, we varied the third axis, the height of the object. With a given major axis and minor axis, increasing the height of the object decreases the curvature along the end points of the contour, without changing the horizontal cross-section (i.e., without changing the correct shape response).

\section{EXPERIMENT 2}

In Experiment 1, it was found that subjects could accurately perceive the 3-D shape of the ellipsoids when the major axis was small, thus producing relatively lower image velocity and lower rates of change in curvature. Our purpose in Experiment 2 was to examine more systematically the importance of image velocity and rate of change in curvature for the shape-recovery process. One variable that affects the image velocity is the extent of the major axis. If the minor axis is constant, to increase the extent of the major axis will increase the image velocity of the contour. Two variables that affect the rate of curvature change are the height of the ellipsoid and the angular rate of rotation. The importance of these variables was assessed in Experiment 2.

An additional goal in Experiment 2 was to produce a more salient 3-D perception of the displays. As stated 
above, 12 of 16 subjects in Experiment 1 reported perceiving the displays as a rotating 3-D object. Informal observations suggested that the presentation of a rotation wherein the major axis became parallel to, and crossed, the line of sight increased the saliency of the rotation-indepth perception. This may be a result of the abrupt changes in contour velocity that occur as the major axis crosses the line of sight. For this reason, the initial and final orientations of the ellipsoid (and thus the orientation of the rotation angle relative to the viewer) were changed in Experiment 2. The subjects were shown displays of ellipsoids that ascillated from an initial orientation of $58^{\circ}$ from the frontal-parallel plane, through the line of sight, to a final position $124^{\circ}$ from the frontalparallel plane.

\section{Method}

Subjects. The subjects were 8 undergraduates from the University of Illinois who were paid for their participation. All subjects were naive regarding the purpose of the study, and all had normal or corrected-to-normal vision.

Design. Four independent variables were examined: the major axis of the ellipsoid, the minor axis of the ellipsoid, the height of the ellipsoid, and the angular velocity of the rotation.

Stimuli. As in Experiment 1, the displays simulated silhouettes of ellipsoids occluding a background of random dots. The contour of the ellipsoid was specified only by occlusion of the background texture. One thousand texture elements were randomly positioned in a background of approximately $388.68 \mathrm{~cm}^{2}$, resulting in a density of 3.1 dots $/ \mathrm{cm}^{2}$. At a viewing distance of $1.7 \mathrm{~m}$, the background subtended a rectangular region of $7.7^{\circ} \times 4.9^{\circ}$ of visual angle, which resulted in 26.5 dots $/ \mathrm{deg}^{2}$. All ellipsoids were presented with the major axis horizontal and were depicted rotating about a vertical axis.

Because the results of Experiment 1 indicated no significant effect for the type of projection, all the displays in Experiment 2 were orthographic projections. In addition, because there was no significant effect of the axis location in Experiment 1, an off-centered rotation was arbitrarily chosen for the displays in Experiment 2.

The displays simulated rotation of ellipsoids from a starting position with the major axis at an angle $58^{\circ}$ from the frontal-parallel plane, to an ending position $124^{\circ}$ from the frontal-parallel plane. The ellipsoids oscillated between these two points, three times for the slower angular velocity condition, and six times for the faster angular velocity condition, thus keeping total display duration constant. The frame duration was $70 \mathrm{msec}$. In the slower angular velocity condition, there were 23 frames with a rotation of $3.0 \%$ frame, resulting in a rotation speed of $7.14 \mathrm{rpm}$. In the faster angular velocity condition, every other frame was displayed, resulting in a rotation of $6.0 \%$ frame and a rotation speed of $14.28 \mathrm{rpm}$. The total display duration was $9.6 \mathrm{sec}$.

The extent of the larger major axis condition was twice that of the smaller major axis condition. The horizontal extent in the larger major axis condition subtended approximately $3.6^{\circ}$ of visual angle at the greatest horizontal extent (major axis $58^{\circ}$ from the perpendicular), and $.5^{\circ}$ at the least extent (with the smallest minor axis; major axis parallel to the line of sight). The horizontal extent in the smaller major axis condition subtended approximately $2.3^{\circ}$ at the greatest extent and $.5^{\circ}$ at the least extent. In the larger major axis condition, the minor to major axis ratios for the three levels of the minor axis were $.08, .16$, and .32 , respectively. In the smaller major axis condition, the ratios were twice these values, at .16, .32 , and .64 , respectively. The height of the ellipsoid for the larger and smaller condition subtended $4.7^{\circ}$ and $2.4^{\circ}$, respectively. The ratios of the major axis to the two levels of height were $1.25: 1$ and
$.625: 1$, for the smaller major axis, and $2.5: 1$ and $1.25: 1$, for the larger major axis. Because all three axes were increased proportionately (by a factor of 2), there were cases wherein the ellipsoids had the same shape, but a difference in scale (for example, the ellipsoid with the larger major axis and height, and middle level of the minor axis, was double the size of the ellipsoid with the three small axes).

Apparatus. The apparatus was the same as that in Experiment 1, with the exception of the viewing tube arrangement. In Experiment 2 , the subjects monocularly viewed the displays through a tube arrangement that limited the field of view to $8.5^{\circ}$ in diameter. The viewing distance to the display was $1.7 \mathrm{~m}$.

Procedure. The procedure was essentially the same as that in Experiment 1. Because the depth responses from Experiment 1 were positively correlated with mean errors for the shape responses (i.e., there was relatively poorer accuracy in the conditions that produced higher depth ratings), depth ratings did not appear to be a useful measure for these displays, and they were dropped for Experiment 2. The subjects were given $\mathbf{2 0}$ min of random practice trials, followed by four presentations of each display condition presented in a random order.

\section{Results and Discussion}

All 8 subjects in Experiment 2 reported perceptions of 3-D objects rotating in depth. Most of the subjects reported oscillation, as depicted, although a few subjects reported that some displays looked like complete $360^{\circ}$ rotations.

The subjects' shape responses (see Figure 7) were analyzed with a four-way ANOVA. The analysis revealed a significant main effect for the minor axis $[F(2,14)=$ $43.3, p<.001]$. There was also a significant main effect of major axis $[F(1,7)=24.8, p<.005]$. The $\omega^{2}$ for these two effects were .49 and .04 , respectively. As expected, the larger major axis condition produced higher (more nearly spherical) responses on the scale. In order to examine accuracy, a $t$ test similar to the analysis in Experiment 1 was conducted on the errors. The result was significant $[t(11)=2.95, p<.05]$, with the larger major axis producing relatively poorer accuracy. It should be noted that the smaller major axis only produced relatively good accuracy with the smaller height condition. As previ-

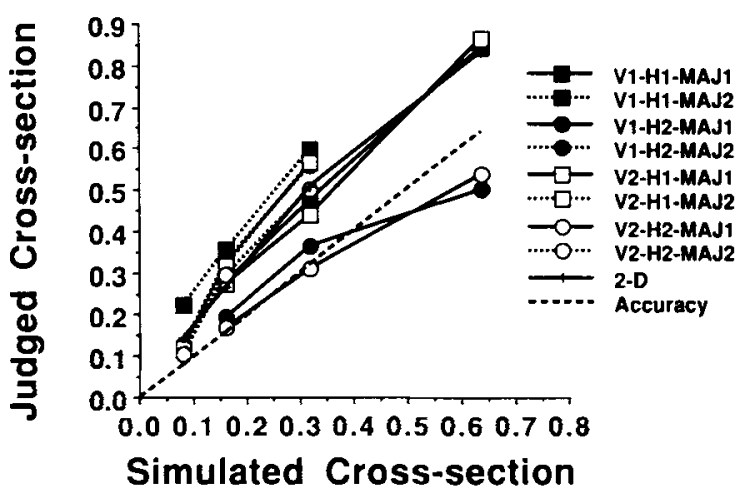

Figure 7. Mean shape judgments as a function of simulated crosssection (minor axis to major axis ratio). In the legend, V1 and V2 refer to the slow and fast angular velocities, respectively. H1 and H2 refer to the small and large heights, and MAJ1 and MAJ2 refer to the small and large major axis conditions, respectively. The dashed line represents perfect performance. 
ously stated, the effect of major axis in these experiments was not merely a function of the eccentricity of the ellipsoid. This can be seen in Figures 5 and 7 if one examines regions of the graphs where the major axis conditions overlap. Comparison of any two points aligned vertically indicates that the larger major axis condition produced poorer accuracy, even though the ratios of the axes were the same. It is important to show that the effects are not merely a function of eccentricity, because there is no a priori reason to believe that the discriminability of ellipsoids under ideal conditions would be constant across the scale. Indeed, an examination of Figure 4 suggests that the ratios near the lower end of the scale might be more discriminable. However, comparison of ellipsoids with similar cross-sectional shapes but different sizes does not reveal equivalent accuracy. One way to provide statistical confirmation of this is to perform a $t$ test across major axis conditions, not matched by the minor axis, as was done previously, but rather matched by shape. There were four pairs of ellipsoids (a pair with Shape 5 and a pair with Shape 9, at each of two levels of velocity) with all three axes retaining the same proportions but differing by a scale factor of 2 . A $t$ test on the errors in shape responses of these pairs was significant $[t(3)=4.08$, $p<.05$ ], demonstrating that the larger ellipsoids were indeed judged to be less elongated than the smaller ellipsoids with the same cross-section.

Contrary to the results of Experiment 1, the variance of curvature from frame to frame was not significantly related to mean error $[r(10)=.12, p>.05]$. This may suggest that subjects' shape responses are not based directly on curvature change. An alternative hypothesis is that curvature change influences the perceived amount of rotation, which in turn influences shape recovery. If this hypothesis is correct, the variance of curvature should be correlated with shape responses whenever the curvature information is used to correctly determine the extent of rotation. This issue was addressed in Experiment 3.

There was a significant effect for height $[F(1,7)=37.0$, $\left.p<.001, \omega^{2}=.05\right]$. Although to increase the height of the object does not change the horizontal cross-section, the taller objects generally produced shape responses indicative of a more horizontally elongated object. One possible reason for this concerns the ambiguity of the 2-D projection (see Figure 2). Elongated, slowly rotating ellipsoids can produce the same transforming 2-D projection as can less elongated, faster rotating ellipsoids. To increase the height of the object decreases the curvature along the end points of the contour. With less curvature change, subjects may perceive less rotation than is actually present, thus producing more elongated shape responses. It should be noted that although the increased height served to lower the scores an average of 2.77 points on the scale, it only had a large effect on accuracy with the smaller major axis condition.

The angular velocity of the ellipsoid had a significant effect as well $[F(1,7)=16.3, p<.005]$, although it accounted for little variance $\left(\omega^{2}=.003\right)$. According to this result, the faster rotating ellipsoids were rated an average of .9 points lower on the shape response scale. However, the effect of velocity here does not appear to be sufficient to account for the wide separation between shape responses in the two major axis conditions, in Experiments 1 and 2. Given the negligible amount of variance accounted for by this variable, image velocity does not appear to be a major factor affecting the recovery of 3-D shape.

The results also showed significant interactions between the major axis and the height $[F(1,7)=9.9, p<.025]$ and between the minor axis and the height $[F(2,24)=$ $6.4, p<.025$ ], although the variance accounted for was small for both effects (.02 and .01 , respectively). With the longer major axis, the height had little effect on the shape responses, which were almost always overestimated by several points on the scale. With the smaller major axis, the increased height lowered the shape responses. It is possible that this again relates to the curvature issue: To increase the height by a given amount has a greater effect on the curvature (along the end points of the contour) when the major axis is relatively small.

The interaction between the minor axis and the height is seen best with the decrease in shape responses that $O c$ curs (see Figure 7) for the larger minor axes in the small major axis, large height conditions. It is interesting that these points, and those of the larger minor axes with the smaller major axis in Experiment 1, were the only conditions that resulted in responses below the line of perfect accuracy, indicating that subjects in only these few cases reported more elongated shapes than were actually presented. If this was due primarily to the subjects' impressions of the lengths of the major axis, then the subjects were actually seeing more depth than was presented in the display. An alternative explanation is that these responses were the result of an underestimation of the minor axis; but this seems unlikely, because the minor axis was present in the 2-D projection of each display used in Experiment 2, and in Experiment 1 the 2-D cues should lead to an overestimation of the minor axis.

For these data points, similar results were obtained across Experiments 1 and 2, which depicted different rotation conditions. Specifically, in Experiment 1, the major axis was approximately specified by the maximum horizontal extent in the image (as the rotation began with the major axis $10^{\circ}$ from the frontal-parallel plane, the projected contour length was $99 \%$ of the major axis). In Experiment 2 , the minor axis was specified by the minimum horizontal extent. In both experiments, use of the maximum and minimum horizontal extents in the image would result in less eccentric (more circular) estimates of the cross-section. Yet the subjects' estimates for these display conditions were more eccentric (more elongated) cross-sections. One possible explanation for this result is that subjects perceived less rotation than was actually simulated. As discussed previously, due to the inherent ambiguity of the transforming 2-D projection, to perceive less rotation than was simulated should lead the subjects to perceive more elongated shapes than were simulated. 


\section{EXPERIMENT 3}

In Experiments 1 and 2, the subjects demonstrated good accuracy in judgments of the cross-section of rotating ellipsoids under certain display conditions. In both experiments, the subjects were more accurate in judging the cross-section in displays with the smaller major axis. In Experiment 2, poorer accuracy occurred in the larger height conditions, suggesting that the reduction in the rate of curvature change affected cross-section estimates. In addition, the subjects in Experiment 2 made shape estimates that were more consistent with the 2-D strategy for the longer major axis. One explanation for this result is that the subjects perceived the rotation of the ellipsoid as a complete $360^{\circ}$ rotation as opposed to an oscillation. Indeed, 3 out of 8 subjects in Experiment 2 indicated during debriefing that some of the objects appeared to undergo a $360^{\circ}$ rotation. The extent of perceived rotation may be a critical variable for the recovery of 3-D shape from deforming contours.

Our purpose in Experiment 3 was to assess the importance of perceived rotation for the shape-recovery process. The subjects were shown displays simulating rotation of ellipsoids of varying shape about a vertical axis. In this experiment, the amount of simulated rotation was varied. This should have an impact on the recovery of shape for two reasons. First, in Experiments 1 and 2, the subjects were presented with displays in which the amount of rotation was restricted. Under those conditions, the displays portrayed only a portion of the total surface area of the ellipsoid. In order to make a correct estimate of the crosssection of the ellipsoid, subjects must interpolate from seen regions of the surface to unseen regions. As the amount of rotation is increased, the subjects are being shown a greater amount of surface area of the ellipsoid, and thus they are required to interpolate a smaller proportion of the total surface.

Second, the failure to correctly determine the crosssection in displays used in Experiments 1 and 2 may have been a result of insufficient information for the perception of rotation in depth. Several possible sources of information (e.g., change in curvature, change in the horizontal extent of the image contour, change in total area of the image contour) may be used for the perception of rotation. An increase in the amount of rotation may result in an increase in the saliency of information for the perception of rotation in depth.

In Experiment 3, the role of perceived extent of rotation in the recovery of 3-D shape was examined. The displays simulated rotation of an ellipsoid through $44^{\circ}, 84^{\circ}$, or $164^{\circ}$. In addition to indicating a shape response, the subjects were required to estimate the amount of rotation simulated in the display. The subjects were presented with a computer display depicting a top view of the rotating object and the viewer's position relative to the object (see Figure 8). They indicated their responses by adjusting, with a mouse, a line (representing the major axis) to indicate the beginning, middle, and end point of rotation, relative to a point representing the viewer. If shape recov-



REMEMBER, THIS IS A TOP VIEW!

Figure 8. The display screen used for the rotation judgments. The diagram represents a top view. Subjects adjusted the line to indicate the beginning, middle, and ending orientation of the major axis of the object during rotation.

ery is dependent on the perception of rotation in depth, then subjects' shape judgments should be related to the amount of perceived rotation.

\section{Method}

Subjects. The subjects were 11 undergraduates from the University of Illinois who were paid for their participation. All subjects were naive regarding the purpose of the study, and all had normal or corrected-to-normal vision.

Design. Three independent variables were examined: the major axis of the ellipsoid, the height of the ellipsoid, and the extent of angular rotation.

Stimuli. As in Experiment 1, the displays simulated silhouettes of ellipsoids occluding a background of random dots. The contour of the ellipsoid was specified only by occlusion of the background texture. One thousand texture elements were randomly positioned in a background of approximately $388.68 \mathrm{~cm}^{2}$, resulting in a density of 3.1 dots $/ \mathrm{cm}^{2}$. At a viewing distance of $1.7 \mathrm{~m}$, the background subtended a rectangular region of $7.7^{\circ} \times 4.9^{\circ}$ of visual angle, which resulted in 26.5 dots $/ \mathrm{deg}^{2}$. All ellipsoids were presented with the major axis horizontal and were depicted rotating about a vertical axis.

The displays were similar to the displays used in Experiment 2, which simulated orthographic projections of opaque ellipsoids rotating about a vertical axis located at an end point. The rotation of the ellipsoid was always symmetrical about the line of sight. The extent of angular rotation was $44^{\circ}, 84^{\circ}$, or $164^{\circ}$, with a rotation speed of $9.5 \mathrm{rpm}$. So that the displays would not appear to be $360^{\circ}$ rotations, the objects did not oscillate. Instead, the objects rotated through the appropriate angle three times, with a $1-\mathrm{sec}$ interval between each rotation. There were 12,22 , and 42 frames for the $44^{\circ}, 84^{\circ}$, and $164^{\circ}$ rotation conditions. The display durations for the $44^{\circ}, 84^{\circ}$, and $164^{\circ}$ rotation conditions were $4.5,6.6$, and $10.8 \mathrm{sec}$, respectively.

The minor axis of the ellipsoid was held constant, whereas the major axis was varied across display conditions. The minor axis to major axis ratios were $.28, .40, .56$, and .72 . The four different major axis conditions subtended, at their greatest extent and for the largest rotation condition, $4.5^{\circ}, 3.1^{\circ}, 2.2^{\circ}$, and $1.7^{\circ}$ visual angles. The height of the ellipsoids was either $2.4^{\circ}$ or $3.6^{\circ}$ of visual angle.

Apparatus. The apparatus and viewing tube arrangement were the same as those in Experiment 2. A Macintosh computer and mouse were used as the response device for the rotation task. 
Procedure. The subjects were instructed that they would see a series of displays of rotating objects. They were told that the displays might appear somewhat spherical, egg-shaped, or flat. These possibilities were demonstrated with the models used in Experiment 1. For each display, the subjects were required to rate the extent of angular rotation, using the Macintosh computer and the mouse. The subjects were presented with a display of a circle, two coordinate axes, and an eye point (see Figure 8). A blinking line, representing the major axis, was adjusted by the subject to indicate the starting point, midpoint, and end point of the rotation. In addition, for each display, the subjects made shape judgments as in Experiment 1, on the 26-point scale depicted in Figure 4. The subjects were told that the display on any trial would be repeated if they wished to see the display again. They were given $20 \mathrm{~min}$ of random practice trials, followed by four presentations of each display condition presented in a random order. At the end of the experiment, the subjects were asked a series of debriefing questions, including, "What did the objects appear to be doing?" They were also asked whether or not they had used any particular strategy to make the shape responses.

\section{Results and Discussion}

Rotation responses. Rotation responses were analyzed in terms of the amount of rotation, and not the actual indication of the beginning and end of the rotation. Generally the subjects were quite consistent in judging the rotation as symmetrical about the line of sight. The mean rotation responses (see Figure 9) for each subject in each condition were analyzed with a three-way ANOVA. There was a significant main effect for the amount of rotation $[F(2,20)=127.9, p<.001]$, in the direction expected. There was a significant main effect for the major axis $[F(3,30)=63.6, p<.001]$. As the major axis increased, the reported amount of rotation also increased. In addition, there was a significant two-way interaction between the rotation and major axis variables $[F(6,60)=10.9, p<$ $.001]$. There were no other significant main effects or interactions. The $\omega^{2}$ values for the main effects of rotation and major axis and for the interaction between rotation and major axis were $.30, .24$, and .04 , respectively.

Shape responses. The mean shape responses for each subject in each condition were analyzed in a three-way ANOVA. There was a significant main effect for the

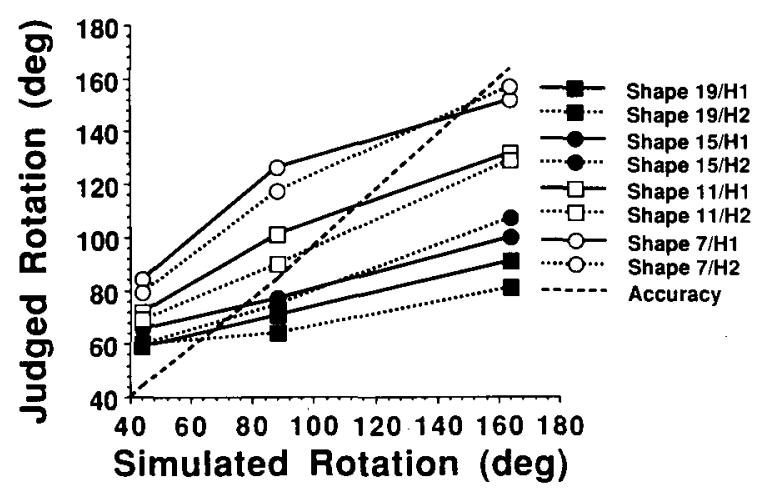

Figure 9. Mean judged rotation as a function of simulated rotation for different shape conditions (see Figure 4 for shape values). Extent of rotation is plotted in degrees. $H 1$ and $H 2$ refer to the small and large height conditions, respectively. The dashed line represents perfect performance. amount of rotation $[F(2,20)=9.2, p<.05]$. The mean shape responses for the three rotation conditions are presented in Figure 10. As the simulated rotation increased, the shape responses decreased, indicating perceptions of more elongated objects. There was a significant main effect for the major axis $[F(3,30)=24.4, p<.01]$, which was expected, since the major axis obviously affects the shape. There was also a significant main effect for the height of the ellipsoid $[F(1,10)=29.5, p<.01]$. This was similar to the result found in Experiment 2; taller objects produced shape responses indicative of more elongated objects. The $\omega^{2}$ values for the main effects of major axis, rotation, and height were $.27, .06$, and .05 , respectively.

There were significant two-way interactions between the height and the major axis $[F(3,30)=3.37, p<.05]$ and between the amount of rotation and the major axis $[F(6,60)=4.41, p<.01]$. There was also a significant three-way interaction among the height, rotation, and major axis variables $[F(6,60)=2.4, p<.05]$. The $\omega^{2}$ values for the two-way interactions between height and major axis and between rotation and major axis, and the threeway interaction among the height, major axis, and rotation conditions were $.003, .011$, and .003 , respectively.

One method of verifying the importance of perceived rotation for the shape-recovery process is to derive an equation in which shape judgments can be predicted from reported rotation. The predicted shapes were derived by considering the greatest horizontal extent of a particular display (at the end of the rotation) and the mean reported rotation for that condition. If the minor axis is held constant (as was done for all of the displays in Experiment 3), one can mathematically relate the size of the major axis to the angular position of the axis and the projected horizontal extent of the axis in the image. For an orthographic projection, the total projected length of a contour of an ellipsoid $(H)$ at an angle $\theta$, where $\theta$ is the angle between the position of the major axis and the projection plane, is given by

$$
H=2 \cdot\left(a^{2} \cdot \cos ^{2} \theta+b^{2} \cdot \sin ^{2} \theta\right)^{1 / 2},
$$

where $a$ is the semimajor axis of the ellipse of the horizontal cross-section, and $b$ is the semiminor axis. If one assumes that the horizontal extent and the semiminor axis are specified in the image, the shape response can be predicted from the reported extent of rotation by solving for the semimajor axis:

$$
a=\left[\left(H^{2} / 4-b^{2} \cdot \sin ^{2} \theta\right) / \cos ^{2} \theta\right]^{1 / 2} .
$$

The correlation between the mean reported shape and the predicted shape was $r(22)=.89, p<.001$, accounting for $79 \%$ of the variance. This correlation was also significant $(p<.05)$ for the individual data from 10 of the 11 subjects $[r(22)=.96, .89, .83, .79, .66, .58, .50, .44$, $.44, .41 ; 1$ subject nonsignificant, $r(22)=-.14]$. This suggests that the extent of perceived rotation was a central factor in the shape estimates provided by the subjects.

Because the perception of rotation in depth was important for the recovery of 3-D shape, an important issue is the information used by subjects to determine the extent 
A

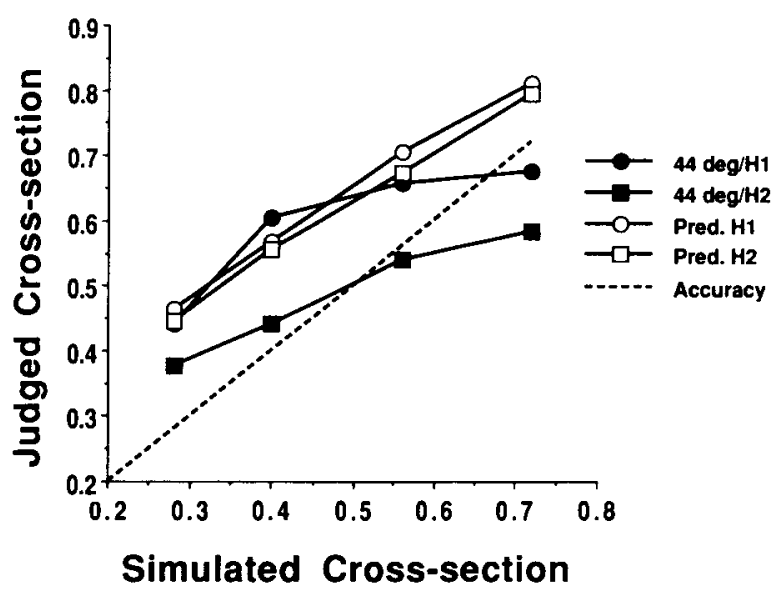

B



C

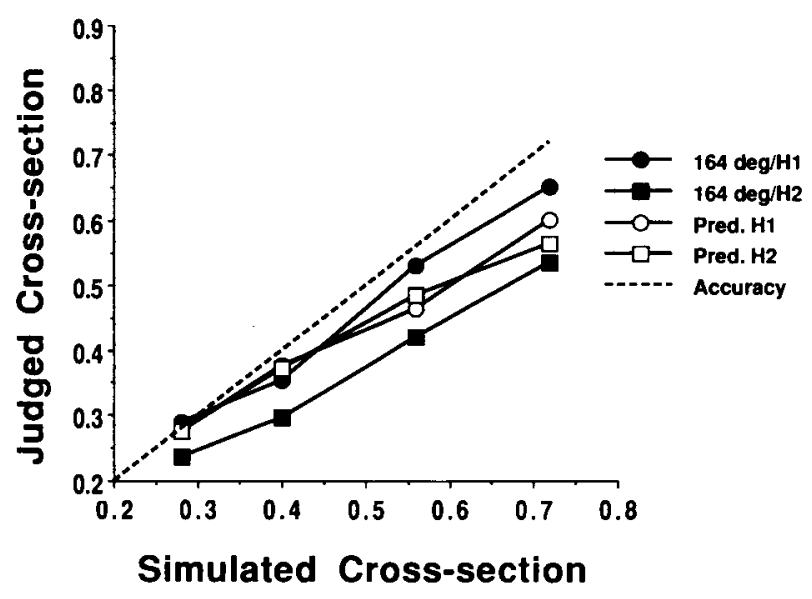

Figure 10. Mean shape judgments, for the $44^{\circ}$ (A), $84^{\circ}(\mathrm{B})$, and $164^{\circ}$ (C) rotation conditions, as a function of simulated cross-section. $\mathrm{H1}$ and $\mathrm{H} 2$ refer to the small and large height conditions, respectively. The rectangles represent the actual responses of the subjects and the triangles represent the predicted responses. The dashed line represents perfect performance. of rotation. One possible source of information is the change in the length of the image contour during rotation. A correlation between the mean reported rotation and the ratio of the maximum horizontal extent to the minimum horizontal extent was $r(10)=.97, p<.001$, accounting for $94 \%$ of the variance. This suggests that the subjects used this deformation of the contour to estimate the extent of angular rotation. It appears that subjects do not use an algorithm that accurately recovers the extent of rotation in deforming contour displays; rather, they rely on a heuristic. The deformation in contour length perpendicular to the axis of rotation may be the best available heuristic. In general, for a given shape, there is a high correlation (.98 or .99) between the ratio of maximum to minimum length and the actual rotation, across a wide range of rotation extents. With a variety of cross-sectional shapes like those in this study, the heuristic is less reliable. The correlation in Experiment 3 between this ratio and the actual rotation was $r(10)=.58$. Interestingly, the regression equation for predicting rotation responses from the ratio of horizontal extents was $R=22.3+39.9 \cdot(\mathrm{max} / \mathrm{min})$, which was similar to the equation for predicting actual rotation from this ratio: $R=24.1+41.4 \cdot(\mathrm{max} / \mathrm{min})$. This suggests that the subjects did nearly as well as they could have done, given that they used this heuristic.

\section{GENERAL DISCUSSION}

The results of these experiments indicate that subjects can, under certain conditions, recover rigid 3-D shape from deforming contours. In the first experiment, the subjects who reported rotation were fairly accurate in correctly identifying the cross-sections of rotating ellipsoids for the smaller major axis conditions. In the second experiment, all subjects reported a perception of a rotating 3-D object, and again they were more accurate for the shorter major axis case. In the third experiment, subjects were fairly accurate in reporting the correct cross-section, provided that they accurately perceived the extent of rotation. If subjects underestimated the extent of rotation, they underestimated the cross-section (i.e., reported a more eccentric cross-section). Likewise, an overestimation of rotation resulted in an overestimation of the cross-section. These results suggest that the extent of rotation is an important source of information for the recovery of 3-D shape from deforming contours. It does not appear that subjects recover 3-D structure from image contours by means of algorithms such as have been used in the artificial intelligence literature (Blake \& Cipolla, 1989; Giblin \& Weiss, 1987).

The reported shape of the ellipsoid was shown to be related to the reported extent of rotation by predicting shape from the estimates of rotation. The mean rotation response from each cell was used, along with the minor axis (which was available in the projection) and the size of the projected contour at the beginning (or end) of the rotation. A correlation between predicted shape and mean reported shape accounted for $79 \%$ of the variance of the shape responses. There was also a correlation of $r(22)=.69, p<.001$, between mean errors in the rotation response and mean 
errors in the shape response. This provides further support for the claim that the perceived extent of angular rotation had a strong effect on shape recovery.

This, of course, raises the question of how subjects recovered the angular rotation. In previous studies, it has been shown that sinusoidal velocity can be used to recover a perception of rotation in depth (see Braunstein, 1977, and Needham \& Andersen, 1989). In the present experiments, this information was not available, because the points that define the image contour did not map to the same locations, over time, on the ellipsoids. The subjects apparently used the maximum and minimum horizontal extents of the image contour to determine the extent of rotation, and this, in turn, influenced the shape-recovery process. An alternative explanation is that the subjects used some heuristic to guess at the shape first, and this then influenced the recovery of rotation. Although this cannot be ruled out, it appears unlikely, because no source of information that we considered (e.g., image velocity, curvature of contour, etc.) accounts for the shape responses as well as the heuristic, discussed earlier, accounts for the rotation judgments. For example, one might suppose that the maximum extent to minimum extent ratio would be the most likely candidate for shape information. However, the reliability of this information for shape recovery is highly dependent on the amount of rotation. This maximum/minimum ratio accounted for $71 \%$ of the variance in the shape responses, as opposed to $94 \%$ of the variance in the rotation responses. Furthermore, other sources of information in the image were not highly correlated with shape responses. One such measure that we tested was the variance in the curvature at the end point of the contour, from frame to frame. This correlation with the mean reported shape was $r(22)=-.497, p<.05$, accounting for only $25 \%$ of the variance of the shape responses. It seems likely, therefore, that the perception of rotation is primary, and that the perceived shape will depend on this recovery of rotation.

This heuristic, involving the change in the extent of the image contour, is a reasonable one that may be used for conditions wherein there is no salient motion correspondence information. This may account for some of the results of previous research on angular velocity discrimination of rotating 3-D shapes. Kaiser (1990) presented subjects with displays simulating pairs of rotating 3-D shapes. The subjects' task was to determine whether or not the angular velocities of the two objects were the same. Kaiser found that differences in the size of the rotating objects affected angular velocity discrimination. These display conditions resulted in differences in the change in contour extent perpendicular to the rotation axis, similar to the changes in the horizontal extent of the image contour in the present study. This suggests that changes in the extent of image contours along a given dimension may be an alternative source of information for determining perceived angular velocity and rotation in depth.

The emphasis in this discussion on the change in length of the contour appears to have relegated the curvature deformation to a minor role. Some pilot work that we conducted is relevant to this point. The objects used in the pilot study were cylindrical solids, with elliptical horizontal cross-sections, but rectangular vertical cross-sections. As these objects rotated about a vertical axis, the contour length deformed in a manner similar to the ellipsoids described in this study. However, the edge of the contour was a vertical line throughout the rotation. Virtually all responses from subjects in this pilot study indicated a nonrigid, 2-D perception. Our tentative conclusion on this issue is that deformation of the curvature of smooth contours is important for the initial perception of rotation in depth, but not for the recovery of the extent of rotation, which apparently is determined by the heuristic described previously. The deformation of curvature of the contour then appears to be analogous to the change in length and direction that Wallach and O'Connell (1953) found important for planar-surfaced solids.

One limitation of these displays should be addressed. Ellipsoids are very singular objects in which the rim is always a planar curve (Koenderink, 1984). An important issue is whether the perceived amount of rotation is a critical factor in the shape-recovery process for objects whose rim is nonplanar. The use of more complex objects with nonplanar rims would, however, introduce additional information (e.g., T-junctions; see Koenderink $\&$ van Doorn, 1976) that might be beneficial to the shaperecovery process.

The recovery of 3-D shape is an important process that is central to many circumstances that require human observers to recognize objects. In the present research, we have examined a new source of information-deforming contours-for this recovery process. We conclude that rotation must be accurately recovered first, in order to determine shape. Further, it was suggested that rotation was recovered from contours by means of a heuristic. It will be the goal of future research to determine whether or not this heuristic generalizes to conditions with salient motion correspondence information. Such a finding would be troublesome for many computational models in which motion correspondence is utilized to derive precise rotation measurements.

\section{REFERENCES}

Andersen, G. J., \& CoRTese, J. M. (1989). 2-D contour perception resulting from kinetic occlusion. Perception \& Psychophysics, 46, 49-55. BIEDERMAN, I. (1985). Human image understanding: Recent research and a theory. Computer Vision, Graphics, \& Image Processing, 32, 29-73. Blake, A., \& Cipolla, R. (1989). Robust estimation of surface curvature from deformation of apparent contours (Tech. Rep. OUEL 1797/89). Oxford, England: University of Oxford.

Braunstein, M. L. (1977). Minimal conditions for the perception of rotary motion. Scandinavian Journal of Psychology, 18, 216-223.

Braunstein, M. L., \& Andersen, G. J. (1984). Shape and depth perception from parallel projections of three-dimensional motion. Journal of Experimental Psychology: Human Perception \& Performance, 10, 749-760.

Braunstein, M. L., Hoffman, D. D., \& Pollick, F. E. (1990). Discriminating rigid from nonrigid motion: Minimum points and views. Perception \& Psychophysics, 47, 205-214. 
Braunstein, M. L., Hoffman, D. D., Shapiro, L. R., Andersen, G. J., BennetT, B. M. (1987). Minimum points and views for the recovery of three-dimensional structure. Journal of Experimental Psychology: Human Perception \& Performance, 13, 335-343.

Giblin, P., \& Weiss, R. (1987). Reconstruction of surfaces from profiles. In Proceedings of the Ist International Conference on Computer Vision (pp. 136-144). London: IEEE.

Hoffman, D. D., Richards, W. A. (1984). Parts of recognition. Cognition, 18, 65-96.

Kaiser, M. K. (1990). Angular velocity discrimination. Perception \& Psychophysics, 47, 149-156.

Kaplan, G. A. (1969). Kinetic disruption of optical texture: The perception of depth at an edge. Perception \& Psychophysics, 6, 193-198.

KoEnderink, J. J. (1984). What does the occluding tell us about solid shape? Perception, 13, 321-330.

KoENDERINK, J. J., * VAN DoORN, A. J. (1976). The singularities of the visual mapping. Biological Cybermetics, 24, 51-59.

LAPPIN, J. S., Doner, J. F., KotTas, B. (1980). Minimal conditions for the visual detection of structure and motion in three dimensions. Science, 209, 717-719.

MetzGer, W. (1934). Tiefenserscheinungen in optischen Bewegungsfeldern. Psychologische Forschung, 20, 195-260.
MILES, W. R. (1931). Movement interpretation of the silhouette of a rotating fan. American Journal of Psychology, 43, 392-405.

NeEdham, A. E., ANDersen, G. J. (1989). Resolving conflicting perceptual interpretations of motion in depth. Investigative Ophthalmology \& Visual Science, 30(Suppl. 3), 251.

PoluCK, F. E. (1989). Shape perception from dynamic occluding contours. Investigative Ophthalmology \& Visual Science, 30(Suppl. 3), 264.

ToDD, J. T. (1984). The perception of three-dimensional structure from rigid and nonrigid motion. Perception \& Psychophysics, 36, 97-103.

TODD, J. T. (1985). Perception of structure from motion: Is projective correspondence of moving elements a necessary condition? Journal of Experimental Psychology: Human Perception \& Performance, 11, 689-710.

Ullman, S. (1979). The interpretation of visual motion. Cambridge, MA: MIT Press.

Wallach, H., O'Connell, D. N. (1953). The kinetic depth effect. Journal of Experimental Psychology, 45, 205-217.

(Manuscript received June 21, 1990; revision accepted for publication December 13, 1990.) 